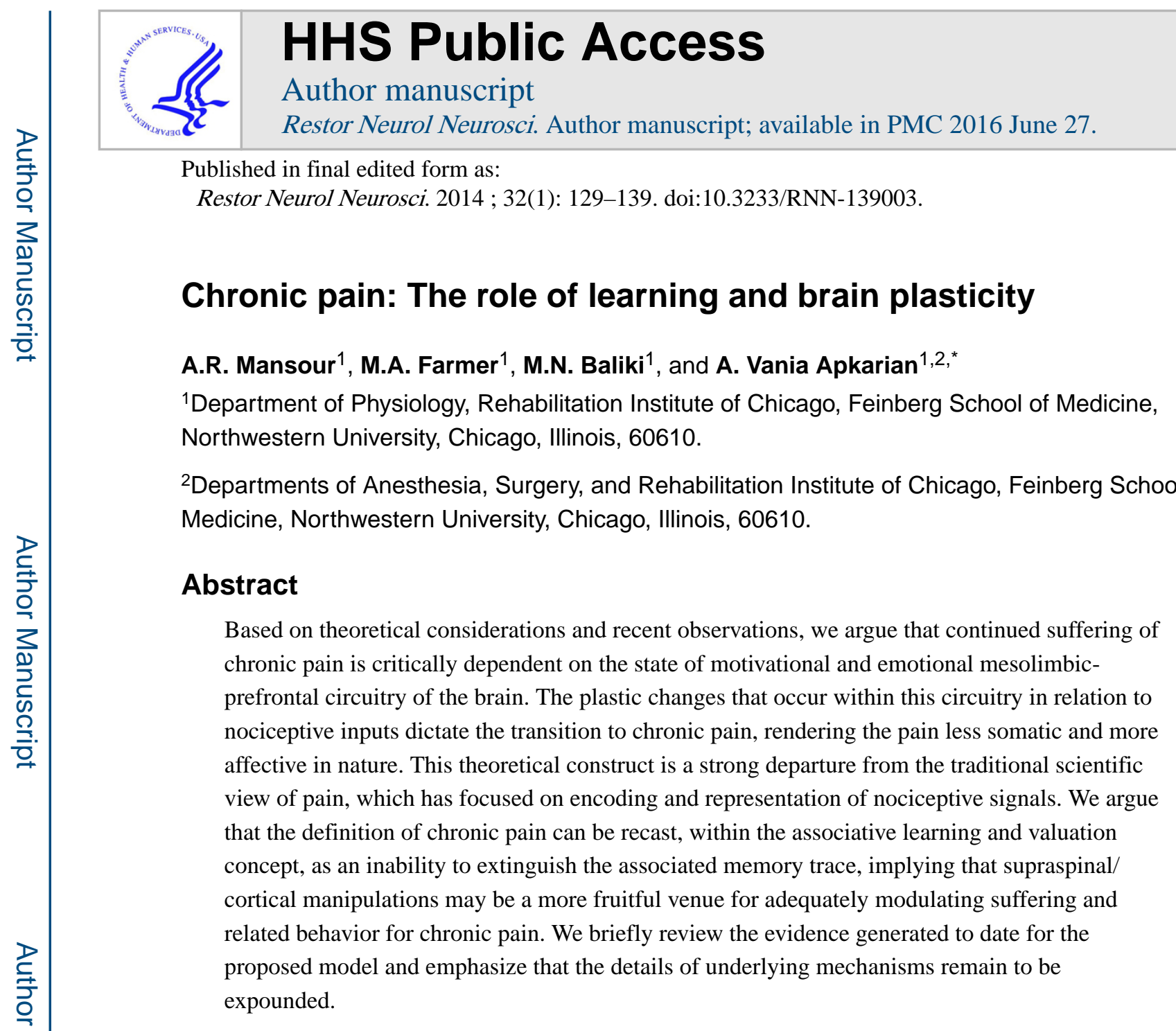

\title{
Introduction
}

In this chapter, we propose a new theory regarding mechanisms of transition to chronic pain, supported by a review of the human and animal studies that are beginning to identify underlying mechanisms of pain chronification. Theoretically, and given recent advances, we argue that the state of the brain's emotional and motivational circuitry, as well as its reorganization following a pain-inciting event, determine the transition to pain chronicity.

The affective associations of chronic pain manifest as increased anxiety, depression, and a dramatically reduced quality of life, as well as other cognitive and behavioral impairments. Clinicians treating chronic pain patients commonly observe that this suffering is maintained even when the intensity of chronic pain is reduced by therapy. It is also a common observation that patients are far more distressed, in disproportion to the pain intensity, about the emotional load associated with chronic pain. Moreover, in many cases the emotional suffering is maintained even though the peripheral signs of the injury, and thus the theoretical source of nociceptive activity, has long disappeared. Indeed, there is ample evidence that drugs that are highly effective in treating acute (and primarily nociceptive)

\footnotetext{
*To whom correspondence should be addressed. a-apkarian@northwestern.edu.
} 
pain, such as non-steroidal anti-inflammatories and opiates, show variable or no effect on treating most chronic pain conditions.

Non-invasive human brain imaging studies have provided the opportunity to directly peer into the brains of chronic pain patients. These studies show no evidence of increased nociceptive representation, but rather they point to enhanced activity in the emotional and motivational cortical-limbic circuitry. Therefore, we propose that understanding and manipulating processes underlying the emotional suffering (cortical-limbic circuitry) should be more successful in treating chronic pain, as compared to the standard approaches that have been tested for decades. These traditional treatment approaches have concentrated on the source of nociceptive signals in the skin and spinal cord, with little success.

Since the work of Pavlov, it has been known that pain is a potent aversive stimulus for creating salient memories. It induces single event learning, with associated memories potentially lasting a lifetime. These concepts have been utilized in the neuroscience of learning and memory for over a century. Surprisingly, they have had little impact on pain research. Human brain imaging studies also point to reorganization (e.g., gray matter atrophy and decreased white matter integrity) of cortical-limbic circuitry that seems to be specific to distinct chronic pain conditions.

We propose that chronic pain is the consequence of plastic changes in cortical-limbic circuitry, leading to new learning and to memory formation that are continuously reinforced and thus cannot be extinguished, as a consequence of the emotional and motivational associations with the painful stimulus, perhaps coupled with enhanced learning abilities due to a predisposition to addictive behavior.

\section{Scale of the problem}

Pain is the primary reason why people seek healthcare. Every day, 50 million Americans are either partially or totally disabled by pain. If untreated, pain can lead to depression, sleeping disorders, immune suppression, eating disorders, cognitive impairment, and other long-term deleterious effects. When pain is the consequence of an acute injury and/or inflammatory process, it may be alleviated through the attenuation of the noxious stimulus or disease processes. However, in modern societies, pain is to a large extent chronic in nature, and despite considerable research efforts, no reproducibly effective therapy for chronic pain has been established to date. According to the Institute of Medicine of the National Academies' recently released report and recommendations on chronic pain (www.iom.edu, released on 6/29/2011), chronic pain affects at least 116 million American adults - more than the total affected by heart disease, cancer, and diabetes combined. Pain costs the nation up to $\$ 635$ billion each year in medical treatment and lost productivity, making "the prevention of pain" the second major priority proposed for the nation's health improvement.

Pain is associated with a negative affective state that contributes to an individual's suffering. Even though European agencies and National Institutes of Health in the United States have funded hundreds of studies on the mechanisms of pain, and despite the fact that these studies have uncovered a wealth of information regarding underlying mechanisms of chronicity, the 
incidence of chronic pain continues to rise and its alleviation remains quixotic, haphazard, and more serendipitous than scientific in nature. At the time of the writing of this chapter, the authors received an email from a New Zealander that epitomizes the sad state of standard chronic pain "management." It states:

"Throughout all the developments and complications [our patient] has experienced with leukemia over the past couple of years, his specialist [Anon] has carefully explained the nature of each problem, outlined the recommended treatment, told us how the drugs work and described the effects it is likely to have. The reliability of his predictions has given us confidence that [our patient's] treatment is based on sound science. By contrast, my initial impression of the medical management of [the patient's] pain was that it was totally unscientific.... they were just throwing drugs at the problem in the hope that something eventually would work."

Currently, the approach to the management of chronic pain is almost exclusively symptombased. Work pioneered in our lab shows that chronic pain impacts the integrity of the cortex, suggesting that it is a progressive and perhaps neurodegenerative disease, the persistence of which should be halted as soon as possible. To this end, the rational path for the prevention and treatment of chronic pain must address the underlying mechanisms of chronicity.

\section{Phantom pain and learning}

Perhaps Patrick D. Wall was the single most emphatic proponent of the idea that pain is strongly modulated by the brain and that cognitive states dramatically influence pain perception. He argued that in children, a mother's kiss was far more effective in alleviating the pain of an acute injury than the use of any analgesic. He also highlighted situations wherein high stress levels masked classical pain perception, despite major injury. Athletes injured in the heat of competition, who only experience pain once the competition is over, serve as a common modern example of this phenomenon. Other, less fortunate, examples are soldiers who rejoice to the news that they are injured as it harbors the opportunity to leave the battlefield (Melzack and Wall, 1986). These examples highlight the fact that pain cannot simply be explained by afferent activity or spinal cord sensitization (i.e., the two most dominant ideas in the field, where the majority of basic science continues to concentrate ((Basbaum et al., 2009; Gold and Gebhart, 2010; Woolf and Salter, 2000).

The classic example of the role of the brain and associated learning mechanisms in pain perception comes from observations and interventions made by Ramachandran some years ago, in patients with phantom pain (Ramachandran and Rogers-Ramachandran, 1996). In his most famous case, a subject who had suffered from phantom pain for over 10 years was trained to use a mirror with which he could induce the illusion of moving the phantom arm. The phantom arm had the associated perception of being stuck in a clenched position, and hence had been a source of phantom pain for years. By observing his intact limb in the mirror and its movement, the subject immediately perceived that the phantom was, in fact, moving. Repeated application of this exercise resulted in the disappearance of the phantom and associated pain. Many others have now confirmed these observations, and the method is used clinically (Rosen and Lundborg, 2005). 
Such cases highlight an unambiguous role of the brain learning circuitry in chronic pain. Given that the pain persists within a nonexistent limb, with the details of the hand and its position vividly represented by the cognitions of the patient, this phantom pain cannot be attributed simply to excessive firings of the injured nerve, given that such firings could not induce such specific spatial patterns of pain location. Moreover, the pain relief with the mirror illusion demonstrates that associative learning, in this case the visual illusion of which the patient is well aware, can permanently counteract a pain that had persisted for many years. The simplest explanation is that associative learning is occurring within the corticolimbic circuitry, most likely with no change in peripheral afferent activity and perhaps due to the unmasking of somatosensory regions due to deafferentation. Therefore, we can conclude that brain learning circuitry, at least in such examples, is a critical part of the pain experience. Even more informative is the fact that both the observation and intervention were generated by a neurologist who is not a pain scientist, and who conceived the intervention based on the principles of associative learning. Moreover, the pain research community continues to ignore this work and its implications, even when some clinics are using the technique for therapy (Rosen and Lundborg, 2005). A recent paper elegantly expounds a full theory for phantom pain and for tinnitus based on these same fundamental tenets and incorporates many elements that we emphasize here as basic components of chronic pain (De Ridder et al., 2011).

In a series of studies almost 30 years ago, Wall, Kaas, Merzenich, and colleagues showed that peripheral nerve injuries in monkeys readily give rise to distortions of sensorimotor somatotopy (Wall et al., 1986). Similar observations were first made by Flor and colleagues in humans, showing that phantom pain is associated with large shifts in body part representation within the cortex (Flor et al., 1995). These observations have resulted in the conclusion, shared in this review, that central rather than peripheral factors may be more critical in pain chronicity (Flor, 2012). The latter was the first evidence of cortical plasticity associated with a chronic pain condition. Since then the evidence for brain plasticity with chronic pain has expanded into the study of brain gray matter and white matter reorganization. New information has continued to be reported across laboratories regarding the relationship and specificity of brain anatomical and functional reorganization across different types of chronic pain (Apkarian et al., 2009; Apkarian et al., 2005).

\section{Pain and learning are intimately related, and chronic pain can be defined in this context}

In a recent review article (Apkarian, 2008), we formulated the relationship between learning and chronic pain:

"Chronic pain is defined as a state of continued suffering, sustained long after the initial inciting injury has healed. In terms of learning and memory one could recast this definition as: Chronic pain is a persistence of the memory of pain and/or the inability to extinguish the memory of pain evoked by an initial inciting injury."

"The novel hypothesis that we advance is that chronic pain is a state of continuous learning, in which aversive emotional associations are continuously made with 
incidental events simply due to the persistent presence of pain. Simultaneously, continued presence of pain does not provide an opportunity for extinction because whenever the subject is re-exposed to the conditioned event he/she is still in pain. Failing to extinguish, therefore, makes the event become a reinforcement of aversive association."

Based on these concepts, we specifically have suggested that the interaction between the prefrontal cortex and brain limbic learning circuitry should be critical for pain to transit from an acute event to a persistent chronic state, as illustrated in Figure 1.

As shown in Figure 1, the overall concept is that nociceptive inputs, which tend to be transient in the healthy organism and thus mainly evoke acute pain perception (illustrated by activation of anterior cingulate, ACC, and insula), would also establish, through the limbic circuitry, learned associations that are gradually extinguished in time. However, when the nociceptive input is more intense and persistent, it can preferentially involve the limbic circuitry, comprised of nucleus accumbens (NAc), amygdala (Amyg), and hippocampus (Hipp), leading to novel learning and memory processes. These synaptic pathways forged by learning in turn interact with the medial and lateral prefrontal cortical circuitry (mPFC, LPFC) and shift cortical activity from a nociceptive perception to more of an emotional suffering state. Additionally, the latter would induce cortical reorganization due to both the coping- and suffering-induced carving of the anatomical and functional organization of the cortex. In this framework, we also conceptualize that descending modulatory pathways may be critically controlled through prefrontal and limbic circuitry, and these systems may in fact even influence the state of excitability of primary afferents, as well as spinal cord neurons. Thus, we propose that, given some predisposing factors, the state of the mesocorticolimbic network determines whether nociceptive inputs become transient or persistent by either preferentially activating extinction pathways or, inversely, by strengthening learning signals that amplify the affective properties of nociceptive inputs. An analogy for this process pertains to the mechanisms related to drug addiction behavior, wherein repeated exposure to an addictive rewarding drug induces an inability to suppress undesired behavior. In the case of chronic pain, pain-related perceptions and behavior become exaggerated and persist through associative learning coordinated by the mesolimbic network. The learning is driven by implicit as well as explicit memories interacting with sub-conscious salience and valuation signals that determine the associative strengths of the remodeled and/or novel synapses. These learning processes interact with coping mechanisms provided by the subject's past experience of their condition. In fact, disparate implicit and explicit associations may underlie the suffering of chronic pain. The model is predicated on an initial nociceptive drive, and the duration and intensity of this drive may be critical to long-term outcomes. The nociceptive drive is usually the consequence of an injury, from varying sources. However, at least in the phantom pain example, one envisions that peripheral afferent activity, or central enhanced activity, drives the formation and maintenance of painrelated cortical memories. In pain conditions where a specific nociceptive site cannot be identified, we still assume that a more diffuse afferent input amplifies the cortical circuitry of pain memories due to a highly potent/sensitized mesolimbic network state. Given these assumptions, the precise source(s) by which pain memories are activated, sustained and/or extinguished are not particularly important, thereby rendering the central distinction between 
inflammatory and neuropathic conditions inconsequential (although the manifestations of each within the spinal cord reorganization remain distinct). Such a framework also provides a continuum of conditions, from acute tissue injury to more diffuse visceral injury, to nerve and CNS injuries, with similar learning-based mechanisms of chronic pain induction through essentially the same mesocorticolimbic system. For a slightly different view regarding the interaction between pain and reward, as well as their contribution to pain chronification, see (Becker et al., 2012).

The main elements comprising the illustrated sub-cortical limbic circuitry can be spatially mapped using a recent meta-analysis tool developed to spatially condense published literature [www.neurosynth.com (Yarkoni et al., 2011)]. Brain activity associated with the term "memory" identifies 1138 published papers and is associated with activity in about $8.4 \%$ of all voxels of the brain (at threshold of $z$-value $>5.0$, which is 5 standard deviations away from baseline activity). The term "reward" identifies 203 studies and activates $1.9 \%$ of the brain, whereas the term "emotion" identifies 326 studies and activates $6 \%$ of the brain. Similarly, the term "pain" identifies 324 studies and activates $6.1 \%$ of the brain. These maps are shown in Figure 2, where we see the primary brain regions involved in each of the terms. We used a high threshold to remove brain regions that would be captured by the studyspecific procedures (for example, at lower thresholds, "memory" seems to include multiple attentional regions simply because of task demands). The specific terms were used to highlight associated limbic structures and display their spatial relationship to activity for "pain". It should be emphasized that these regions are not exclusively activated for these terms alone (e.g., emotion, memory, reward), and all three sub-cortical limbic networks are involved in aspects of emotional learning and memory. Yet, the robust spatial differences between terms also identify the core structures associated with each, at a very high statistical confidence. It is also important to emphasize that the term "pain" primarily captures brain imaging studies of acute painful stimuli applied to healthy subjects, and in fact a deviation away from this activity pattern is what we claim creates a chronic pain condition.

Below we briefly review the accumulating evidence that supports the model outlined in Figure 1, which hinges on the limbic circuitry demonstrated in Figure 2. Brain activity for chronic pain shifts away from the pattern shown for "pain" in Figure 2 to more prefrontal and limbic circuitry, as indicated in recent reviews (Apkarian et al., 2011; Farmer et al., 2012). We emphasize that the model remains a general template without predefined mechanistic details. This vagueness is intentionally preserved, as many details remain to be understood and addressed in the future, and they may deviate from traditional assumptions of acute aversive learning.

\section{Pain perception as a brain dynamical state}

Human brain imaging studies have yet to identify a single cortical voxel specifically dedicated to nociception. The most ardent proponent of a lack of specificity of cortical activity for pain has recently been advanced by Iannetti, who presents intriguing evidence for his claims. Iannetti even challenges the idea that laser evoked cortical potentials are uniquely related to pain perception, in contrast to simply reflecting a salience detection signal (Wang et al., 2010). Instead, we conceptualize pain perception as the product of 
network interactions between brain regions exchanging and processing incoming nociceptive inputs. No matter how modest the stimulus intensity, acute painful stimuli seem to activate about $10 \%$ of the cortical mantle (Figure 2), which roughly translates to 8-10 billion neurons. Note that, in contrast, the number of nociceptive neurons identified in the primate brain over the last 50 years is less than 100 !

Recently, we have expounded on the view of pain perception as a brain dynamical state (Farmer et al., 2012). The main emphasis of this viewpoint is that interactions between brain regions must be incorporated in the field's attempts to disambiguate cognitive states. Brain oscillatory activity in and of itself provides important information regarding such interactions (Baliki, Baria, et al., 2011; Baria et al., 2011). Brain resting state studies provide the means of elucidating network interactions in the absence of external drives. Multiple studies now show abnormal network properties for various chronic pain conditions. Thus, chronic pain cannot be considered a unitary entity but a conglomeration of unique brain states, the details of which dictate the specific properties of each type of clinical pain and the relative extent to which any given condition may be ruminative, depressive, etc, as dictated by the relative involvement of the elements of the mesolimbic circuitry.

\section{Overview of hints of mechanisms for the transition from acute to chronic pain}

Over the last 10 years, Apkarian's group has pioneered the development of brain imaging methods that can be specifically used to study brain properties of chronic pain. A large portion of this work targets chronic back pain (CBP) brain. We published the first study demonstrating that cortical grey matter density decreases regionally in CBP (Apkarian, Sosa, Sonty, et al., 2004). Since this study, > 50 studies have similarly described brain morphological changes across various chronic pain conditions. We argued that this pattern of changes in brain morphometry may be related to the shift in CBP pain perception from sensory (nociceptive) to emotional (hedonic) areas of the brain. This hypothesis was corroborated by our evidence that CBP patients exhibit impaired emotional decision making in proportion to the magnitude of their back pain (Apkarian, Sosa, Krauss, et al., 2004), implying that the emotionally salient nature of the back pain interferes with other emotional tasks. This hypothesis was further supported using functional imaging, whereby we have sought to characterize the actual pain experienced individuals with back pain by identifying brain regions related to the fluctuations of spontaneous (un-provoked) back pain. This approach yielded the novel finding that the spontaneous pain of CBP engages $\mathrm{mPFC}$, a brain region that modulates emotional evaluation relative to the self (Baliki et al., 2006).

Furthermore, we were able to experimentally demonstrate a double-dissociation between acute thermal pain applied to the back and spontaneous back pain representations in the brain, with the former encoded primarily in the insula and the latter in the mPFC. More recently, we have shown that brain activity elicited by thermal pain is identical between healthy controls and CBP patients, in terms of the brain areas that encode an acute painful stimulus or its perception are concerned. The only difference in brain activity between the two groups related to the bilateral NAc. We observed a NAc salience signal at the onset of painful thermal stimuli, as well as an analgesia-related reward signal at stimulus offset. This 
analgesia-related reward signal was reversed in direction in CBP, indicating abnormal valuation of acute pain relief. We further demonstrated that the strength of functional connectivity between $\mathrm{mPFC}$ and NAc was proportional to the magnitude of CBP back pain (Baliki et al., 2010).

These results, together with our fMRI studies in other chronic pain conditions (Baliki et al., 2008; Baliki et al., 2005; Farmer et al., 2011; Geha et al., 2007; Geha, Baliki, Wang, et al., 2008) and our additional brain morphometry studies (Baliki, Schnitzer, et al., 2011; Geha, Baliki, Harden, et al., 2008), have prompted the proposal of a new mechanistic model for the transition to pain chronicity. This model was proposed and expounded in three review articles (Apkarian, 2008; Apkarian et al., 2009; Apkarian et al., 2011). It proposes that learning mechanisms within the limbic circuitry give rise to the transition from acute to chronic pain and render the pain more emotional (Figures 1 and 2). Within the context of this model we undertook a longitudinal observational brain imaging study, wherein subacute back pain (SBP) patients are tracked over a year as they transition to either persistent pain (SBPp; i.e., chronicity) or into recovery (SBPr), thereby enabling comparisons of brain parameters in this time window and in contrast to healthy and CBP patients (Baliki et al., 2012). We observed that gray matter density decreased only in SBPp patients, yet this is a slow process that is preceded by functional connectivity differences detectable at the first brain scan session. Therefore the functional connectivity strength at this baseline distinguished between SBPp and SBPr $\left(\mathrm{p}<10^{-3}\right)$ and PREDICTED the groupings at high accuracy ( $81 \%$ one year from scan 1$)$. This is the first time that a specific brain circuit in humans pinpoints the transition from acute to chronic back pain. The identified circuit is fully consistent with our earlier human brain imaging studies, our earlier studies in rodents (Centeno et al., 2009; Metz et al., 2009; Millecamps et al., 2007), our proposed model, our preliminary brain imaging data in rodents, and recent studies of rodents in other labs (Gear and Levine, 2011; Johansen and Fields, 2004; Qu et al., 2011; Sarkis et al., 2011). This subacute propensity result complements our proposed model, as it identifies the more critical elements that mediate transition to chronicity.

\section{Abnormal learning/forgetting and the role of the hippocampus in chronic pain}

The hippocampus is considered the primary brain structure for storage and retrieval of longterm explicit memories. It is also extensively implicated in emotional stress conditions, like anxiety and depression. Moreover, specific types of learning paradigms seem to require the hippocampus. The science of pain research has for the most part ignored the involvement of the hippocampus in pain perception or pain behavior. This is likely due to a lack of convincing nociceptive projections to the structure, and there is no electrophysiological evidence for nociceptive responses within the region (however, it is unclear how rigorous the search for hippocampal nociceptive neurons has been).

As chronic pain patients display a variety of cognitive abnormalities, and given that we have hypothesized that conditional learning should be impaired with chronic pain, we have sought evidence for the latter in animal models of neuropathic pain. For a number of years, we used 
classical conditioning acquisition and extinction paradigms in multiple rodent models of neuropathic pain, and admittedly, we were rather confused with the outcomes. While with some experimenters we would obtain evidence of a lack of extinction in neuropathic animals, and in other experiments, we could not replicate the results. Finally Mutso, in collaboration with Radulovic, resolved the problem (Mutso et al., 2012). It turns out that conditioned learning and extinction, when induced for cues, is normal. However, when induced for a specific context, extinction is dramatically inhibited in rodents with neuropathic injuries. The observation is important given that contextual learning requires an intact hippocampus. Thus the study indicates a specific hippocampal learning deficit. Mutso et al. were also able to show physiological, molecular, and neurogenesis abnormalities in the hippocampus of neuropathic injury rodents (Mutso et al., 2012). Furthermore, they provided evidence that at least some of the abnormalities were dependent on the pain itself, rather than on the general heightened anxiety that accompanies persistent pain. Additionally, they observed that in humans with chronic pain, the hippocampal volume is decreased, presumably due to some of the same mechanisms observed in the rodent. Observations consistent with these results have been recently reported (Johnston et al., 2011; Ren et al., 2011).

\section{Amygdala, descending modulation, and learning}

Extensive evidence points to the fact that the amygdala is critical for learning. Studies in rats and humans indicate that glucocorticoid effects on memory consolidation are mediated through noradrenergic activation of the basolateral amygdala, as well as through interactions of the basolateral amygdala with other brain regions. Furthermore, memory retrieval and working memory performance are impaired with high circulating levels of glucocorticoids (Roozendaal et al., 2006). Thus, one would expect that amygdala- mediated learning and memory should be abnormal in chronic pain. To our knowledge, this has not been directly tested in humans. On the other hand, there is extensive evidence for the involvement of the amygdala in animal models of chronic pain; for example, neurons in the region become hyperexcited, thereby influencing dorsal horn neuronal excitability and changing the interaction between the amygdala and the medial prefrontal cortex (Ji et al., 2010; Neugebauer et al., 2004). The evidence for involvement of the amygdala in chronic pain in human neuroimaging studies is minimal. The reason for this omission is most likely technical. Given that the human amygdala is located at the interface between the brain and CSF, it is highly susceptible to magnetic resonance artifacts. Also, given its location, standard brain registration and motion correction approaches tend to distort the region. Therefore, special attention is required to study amygdala function in humans with chronic pain.

Descending modulation has long been demonstrated to be involved in controlling the gain of nociceptive afferent inputs into the spinal cord. More recent evidence demonstrated that descending modulation may play a critical role in the maintenance of central sensitization in neuropathic injured animals (Vera-Portocarrero et al., 2006; Zhang et al., 2009). As the prefrontal cortical circuitry, as well as subcortical limbic circuitry, impinge on descending modulatory pathways, it is reasonable to assume that the effects of descending modulation on the spinal cord activity reflects various states of the interactions of these supraspinal 
circuitry. This in turn implies that distorted leaning and memory processes may be influencing the spinal cord responses to nociceptive afferents.

\section{Functional implications of underlying circuitry}

The brain circuitry identified as critical for pain chronification can be cast within the rubric of appetitive and aversive motivational learning and memory formation. As pain provides a teaching signal that enables individuals to avoid future harm (Apkarian, 2008; Johansen and Fields, 2004), it is a primary punisher and its relief gives rise to negative reinforcement. Motivational information provided by nociceptive inputs should contribute to the activity of circuitry involved in predicting the utility and costs of competing goals, and to behavioral decisions in the presence of conflict (Fields, 2006; Glimcher, 2003; Glimcher et al., 2009; Rolls, 2005). Neural mechanisms of reward valuation and appetitive motivation engage NAc, ventral tegmental area (VTA), and PFc (Goeders and Smith, 1983). Furthermore, both dopaminergic projections from VTA to the NAc and to the cortex, as well as glutamatergic inputs to the NAc from the amygdala, hippocampus, and PFc, collectively comprise the mesolimbic-prefrontal circuit (Ambroggi et al., 2008; Carlezon and Thomas, 2009), which is critical in appetitive behaviors instructed by conditioned cues. Accumulating evidence by us, and others, now shows that this system is also engaged with pain, its salience, and its negative reinforcement value (Baliki et al., 2010; Becerra et al., 2001; Gear and Levine, 2011; Sarkis et al., 2011; Seymour et al., 2004; Seymour et al., 2005). Moreover, we show that the responses of this system to painful stimuli are distorted in chronic pain (Baliki et al., 2010), and that connectivity between mPFC and NAc predicts transition to chronic pain (Baliki et al., 2012).

Dysfunction of the mesolimbic-prefrontal network is a hallmark of addiction, where the corticostriatal circuit is a sub-portion of this circuit. Moreover, all substances of abuse selfadministered by humans that can result in addiction are believed to exert their reinforcing effects by increasing DA in NAc (Koob, 1992). The persistent nature of addiction is associated with activity-induced plasticity of neurons within VTA and NAc, dysfunction of $\mathrm{PFc}$, long-term down-regulation of DA receptors and DA production, as well as enhanced glutamatergic transmission from PFc to NAc (Volkow et al., 2011). Given that we identify the main components of this same circuitry in pain chronification, we assume close parallels between the mechanisms leading to addiction and to pain chronification. We therefore propose that transition to chronic pain is dependent on activity-induced plasticity of the mesolimbic-prefrontal circuit, leading to reorganization of the neocortex. Thus, similar to addiction, chronic pain may be viewed as a brain disease state, but in this case initiated by peripheral nociceptors, and either extinguished or maintained by factors predisposing the extent to which the mesolimbic emotional learning circuitry reacts to the inciting event. The specific parameters controlling the mesolimbic response and the reorganization of this circuitry (for example the extent to which it shares properties observed in drug addiction) remain to be elucidated. Moreover, the extent to which chronic pain highjacks brain reward/ addiction circuitry, or uniquely and in a parallel fashion reorganizes components of this network remain to be studied. 
Overall, we put forward the general idea that, in contrast to acute pain, chronic pain is not a unitary concept. Various kinds of chronic pain are presented by specific brain functional and anatomical profiles, even though verbally subjects continue to dub all of them pain states. In contrast to the agnostic definition of "pain that persists past the healing process", we redefine chronic pain as pain that does not extinguish its memory trace. The latter definition assumes the critical role of mesocorticolimbic circuitry in the control of pain chronification, which we assume shares many of the neurobiological mechanistic properties that have been observed in drug addiction. This radical departure, from the dominant view based primarily on the general approach that afferent activity and spinal cord circuits are sufficient to understand chronic pain, does not deny the respective contribution of peripheral and spinal cord processes in chronic pain but expands the idea by placing the brain emotional learning and memory circuitry as central to an adequate understanding of chronic pain.

\section{Acknowledgments}

We thank the Apkarian lab members who have contributed to the large sets of data collected and analyzed over the last years. Also, we thank all patients and healthy subjects that have taken part in these studies. The National Institutes of Health NINDS has continuously funded the human and animal studies in Apkarian's lab.

\section{References}

Ambroggi F, Ishikawa A, Fields HL, Nicola SM. Basolateral amygdala neurons facilitate rewardseeking behavior by exciting nucleus accumbens neurons. Neuron. 2008; 59(4):648-661. [PubMed: 18760700]

Apkarian AV. Pain perception in relation to emotional learning. Curr Opin Neurobiol. 2008; 18(4): 464-468. [PubMed: 18835354]

Apkarian AV, Baliki MN, Geha PY. Towards a theory of chronic pain. Prog Neurobiol. 2009; 87(2):8197. [PubMed: 18952143]

Apkarian AV, Bushnell MC, Treede RD, Zubieta JK. Human brain mechanisms of pain perception and regulation in health and disease. Eur J Pain. 2005; 9(4):463-484. [PubMed: 15979027]

Apkarian AV, Hashmi JA, Baliki MN. Pain and the brain: Specificity and plasticity of the brain in clinical chronic pain. Pain. 2011; 152(3):s49-s64. [PubMed: 21146929]

Apkarian AV, Sosa Y, Krauss BR, Thomas PS, Fredrickson BE, Levy RE, Harden RN, Chialvo DR. Chronic pain patients are impaired on an emotional decision-making task. Pain. 2004; 108:129-136. [PubMed: 15109516]

Apkarian AV, Sosa Y, Sonty S, Levy RE, Harden RN, Parrish TB, Gitelman DR. Chronic back pain is associated with decreased prefrontal and thalamic gray matter density. J Neurosci. 2004; 24:10410 10415. [PubMed: 15548656]

Baliki MN, Baria AT, Apkarian AV. The cortical rhythms of chronic back pain. J Neurosci. 2011; 31(39):13981-13990. [PubMed: 21957259]

Baliki MN, Chialvo DR, Geha PY, Levy RM, Harden RN, Parrish TB, Apkarian AV. Chronic pain and the emotional brain: Specific brain activity associated with spontaneous fluctuations of intensity of chronic back pain. J Neurosci. 2006; 26(47):12165-12173. [PubMed: 17122041]

Baliki MN, Geha PY, Fields HL, Apkarian AV. Predicting value of pain and analgesia: Nucleus accumbens response to noxious stimuli changes in the presence of chronic pain. Neuron. 2010; 66(1):149-160. [PubMed: 20399736]

Baliki MN, Geha PY, Jabakhanji R, Harden N, Schnitzer TJ, Apkarian AV. A preliminary fMRI study of analgesic treatment in chronic back pain and knee osteoarthritis. Mol Pain. 2008; 4:47. [PubMed: 18950528]

Baliki MN, Katz J, Chialvo DR, Apkarian AV. Single subject pharmacological-MRI (phMRI) study: Modulation of brain activity of psoriatic arthritis pain by cyclooxygenase- 2 inhibitor. Mol Pain. 2005; 1:32. [PubMed: 16266429] 
Baliki MN, Petre B, Torbey S, Herrmann KM, Huang L, Schnitzer TJ, Fields HL, Apkarian AV. Corticostriatal functional connectivity predicts transition to chronic back pain. Nat Neurosci. 2012; 15(8):1117-1119. [PubMed: 22751038]

Baliki MN, Schnitzer TJ, Bauer WR, Apkarian AV. Brain morphological signatures for chronic pain. PLoS One. 2011; 6(10):e26010. [PubMed: 22022493]

Baria AT, Baliki MN, Parrish T, Apkarian AV. Anatomical and functional assemblies of brain BOLD oscillations. J Neurosci. 2011; 31(21):7910-7919. [PubMed: 21613505]

Basbaum AI, Bautista DM, Scherrer G, Julius D. Cellular and molecular mechanisms of pain. Cell. 2009; 139(2):267-284. [PubMed: 19837031]

Becerra L, Breiter HC, Wise R, Gonzalez RG, Borsook D. Reward circuitry activation by noxious thermal stimuli. Neuron. 2001; 32(5):927-946. [PubMed: 11738036]

Becker S, Gandhi W, Schweinhardt P. Cerebral interactions of pain and reward and their relevance for chronic pain. Neurosci Lett. 2012; 520(2):182-187. [PubMed: 22440855]

Carlezon WA Jr, Thomas MJ. Biological substrates of reward and aversion: a nucleus accumbens activity hypothesis. Neuropharmacology. 2009; 56(Suppl 1):122-132. [PubMed: 18675281]

Centeno MV, Mutso A, Millecamps M, Apkarian AV. Prefrontal cortex and spinal cord mediated antineuropathy and analgesia induced by sarcosine, a glycine-T1 transporter inhibitor. Pain. 2009; 145(1-2):176-183. [PubMed: 19577367]

De Ridder D, Elgoyhen AB, Romo R, Langguth B. Phantom percepts: Tinnitus and pain as persisting aversive memory networks. Proc Natl Acad Sci U S A. 2011; 108(20):8075-8080. [PubMed: 21502503]

Farmer MA, Baliki MN, Apkarian AV. A dynamic network perspective of chronic pain. Neurosci Lett. 2012; 520(2):197-203. [PubMed: 22579823]

Farmer MA, Chanda ML, Parks EL, Baliki MN, Apkarian AV, Schaeffer AJ. Brain functional and anatomical changes in chronic prostatitis/chronic pelvic pain syndrome. J Urol. 2011; 186(1):117124. [PubMed: 21571326]

Fields, HL. Proceedings of the 11th world congress on pain. Seattle: IASP press; 2006. A motivationdecision model of pain: The role of opioids; p. 449-459.

Flor H. New developments in the understanding and management of persistent pain. Curr Opin Psychiatry. 2012; 25(2):109-113. [PubMed: 22227632]

Flor H, Elbert T, Knecht S, Wienbruch C, Pantev C, Birbaumer N, Larbig W, Taub E. Phantom-limb pain as a perceptual correlate of cortical reorganization following arm amputation. Nature. 1995; 375(6531):482-484. [PubMed: 7777055]

Gear RW, Levine JD. Nucleus accumbens facilitates nociception. Exp Neurol. 2011; 229(2):502-506. [PubMed: 21458450]

Geha PY, Baliki MN, Chialvo DR, Harden RN, Paice JA, Apkarian AV. Brain activity for spontaneous pain of postherpetic neuralgia and its modulation by lidocaine patch therapy. Pain. $2007 ; 128(1-2)$ : 88-100. [PubMed: 17067740]

Geha PY, Baliki MN, Harden RN, Bauer WR, Parrish TB, Apkarian AV. The brain in chronic CRPS pain: Abnormal gray-white matter interactions in emotional and autonomic regions. Neuron. 2008; 60(4):570-581. [PubMed: 19038215]

Geha PY, Baliki MN, Wang X, Harden RN, Paice JA, Apkarian AV. Brain dynamics for perception of tactile allodynia (touch-induced pain) in postherpetic neuralgia. Pain. 2008; 138(3):641-656. [PubMed: 18384958]

Glimcher, PW. Decisions, uncertainty, and the brain: The science of neuroeconomics. Cambridge: MIT press; 2003.

Glimcher, PW.; Camerer, CF.; Fehr, E.; Poldrack, RA. Neuroeconomics. London: Elsevier; 2009.

Goeders NE, Smith JE. Cortical dopaminergic involvement in cocaine reinforcement. Science. 1983; 221(4612):773-775. [PubMed: 6879176]

Gold MS, Gebhart GF. Nociceptor sensitization in pain pathogenesis. Nat Med. 2010; 16(11):12481257. [PubMed: 20948530] 
Ji G, Sun H, Fu Y, Li Z, Pais-Vieira M, Galhardo V, Neugebauer V. Cognitive impairment in pain through amygdala-driven prefrontal cortical deactivation. J Neurosci. 2010; 30(15):5451-5464. [PubMed: 20392966]

Johansen JP, Fields HL. Glutamatergic activation of anterior cingulate cortex produces an aversive teaching signal. Nat Neurosci. 2004; 7(4):398-403. [PubMed: 15004562]

Johnston IN, Maier SF, Rudy JW, Watkins LR. Post-conditioning experience with acute or chronic inflammatory pain reduces contextual fear conditioning in the rat. Behav Brain Res. 2011; 226(2): 361-368. [PubMed: 21920390]

Koob GF. Neural mechanisms of drug reinforcement. Ann N Y Acad Sci. 1992; 654:171-191. [PubMed: 1632582]

Melzack, R.; Wall, PD. The challenge of pain. Harmondsworth: Penguin; 1986.

Metz AE, Yau HJ, Centeno MV, Apkarian AV, Martina M. Morphological and functional reorganization of rat medial prefrontal cortex in neuropathic pain. Proc Natl Acad Sci U S A. 2009; 106(7):2423-2428. [PubMed: 19171885]

Millecamps M, Centeno MV, Berra HH, Rudick CN, Lavarello S, Tkatch T, Apkarian AV. Dcycloserine reduces neuropathic pain behavior through limbic NMDA-mediated circuitry. Pain. 2007; 132(1-2):108-123. [PubMed: 17449176]

Mutso AA, Radzicki D, Baliki MN, Huang L, Banisadr G, Centeno MV, Radulovic J, Martina M, Miller RJ, Apkarian AV. Abnormalities in hippocampal functioning with persistent pain. J Neurosci. 2012; 32(17):5747-5756. [PubMed: 22539837]

Neugebauer V, Li W, Bird GC, Han JS. The amygdala and persistent pain. Neuroscientist. 2004; 10(3): 221-234. [PubMed: 15155061]

Qu C, King T, Okun A, Lai J, Fields HL, Porreca F. Lesion of the rostral anterior cingulate cortex eliminates the aversiveness of spontaneous neuropathic pain following partial or complete axotomy. Pain. 2011; 152(7):1641-1648. [PubMed: 21474245]

Ramachandran VS, Rogers-Ramachandran D. Synaesthesia in phantom limbs induced with mirrors. Proc Biol Sci. 1996; 263(1369):377-386. [PubMed: 8637922]

Ren WJ, Liu Y, Zhou LJ, Li W, Zhong Y, Pang RP, Xin WJ, Wei XH, Wang J, Zhu HQ, Wu CY, Qin ZH, Liu G, Liu XG. Peripheral nerve injury leads to working memory deficits and dysfunction of the hippocampus by upregulation of TNF-alpha in rodents. Neuropsychopharmacology. 2011; 36(5):979-992. [PubMed: 21289602]

Rolls, ET. Emotion explained. Oxford: Oxford University Press; 2005.

Roozendaal B, Okuda S, de Quervain DJ, McGaugh JL. Glucocorticoids interact with emotion-induced noradrenergic activation in influencing different memory functions. Neuroscience. 2006; 138(3): 901-910. [PubMed: 16310958]

Rosen B, Lundborg G. Training with a mirror in rehabilitation of the hand. Scand J Plast Reconstr Surg Hand Surg. 2005; 39(2):104-108. [PubMed: 16019738]

Sarkis R, Saade N, Atweh S, Jabbur S, Al-Amin H. Chronic dizocilpine or apomorphine and development of neuropathy in two rat models I: behavioral effects and role of nucleus accumbens. Exp Neurol. 2011; 228(1):19-29. [PubMed: 21146525]

Seymour B, O'Doherty JP, Dayan P, Koltzenburg M, Jones AK, Dolan RJ, Friston KJ, Frackowiak RS. Temporal difference models describe higher-order learning in humans. Nature. 2004; 429(6992): 664-667. [PubMed: 15190354]

Seymour B, O'Doherty JP, Koltzenburg M, Wiech K, Frackowiak R, Friston K, Dolan R. Opponent appetitive-aversive neural processes underlie predictive learning of pain relief. Nat Neurosci. 2005; 8(9):1234-1240. [PubMed: 16116445]

Vera-Portocarrero LP, Zhang ET, Ossipov MH, Xie JY, King T, Lai J, Porreca F. Descending facilitation from the rostral ventromedial medulla maintains nerve injury-induced central sensitization. Neuroscience. 2006; 140(4):1311-1320. [PubMed: 16650614]

Volkow ND, Wang GJ, Fowler JS, Tomasi D, Telang F, Baler R. Addiction: decreased reward sensitivity and increased expectation sensitivity conspire to overwhelm the brain's control circuit. Bioessays. 2011; 32(9):748-755. [PubMed: 20730946] 
Wall JT, Kaas JH, Sur M, Nelson RJ, Felleman DJ, Merzenich MM. Functional reorganization in somatosensory cortical areas $3 \mathrm{~b}$ and 1 of adult monkeys after median nerve repair: Possible relationships to sensory recovery in humans. J Neurosci. 1986; 6(1):218-233. [PubMed: 3944620]

Wang A, Mouraux A, Liang M, Iannetti GD. Stimulus novelty, and not neural refractoriness, explains the repetition suppression of laser-evoked potentials (LEPs). J Neurophysiol. 2010; 104(4):21162124. [PubMed: 20592123]

Woolf CJ, Salter MW. Neuronal plasticity: Increasing the gain in pain. Science. 2000; 288(5472): 1765-1769. [PubMed: 10846153]

Yarkoni T, Poldrack RA, Nichols TE, Van Essen DC, Wager TD. Large-scale automated synthesis of human functional neuroimaging data. Nat Methods. 2011; 8(8):665-670. [PubMed: 21706013]

Zhang W, Gardell S, Zhang D, Xie JY, Agnes RS, Badghisi H, Hruby VJ, Rance N, Ossipov MH, Vanderah TW, Porreca F, Lai J. Neuropathic pain is maintained by brainstem neurons coexpressing opioid and cholecystokinin receptors. Brain. 2009; 132(Pt 3):778-787. [PubMed: 19050032] 


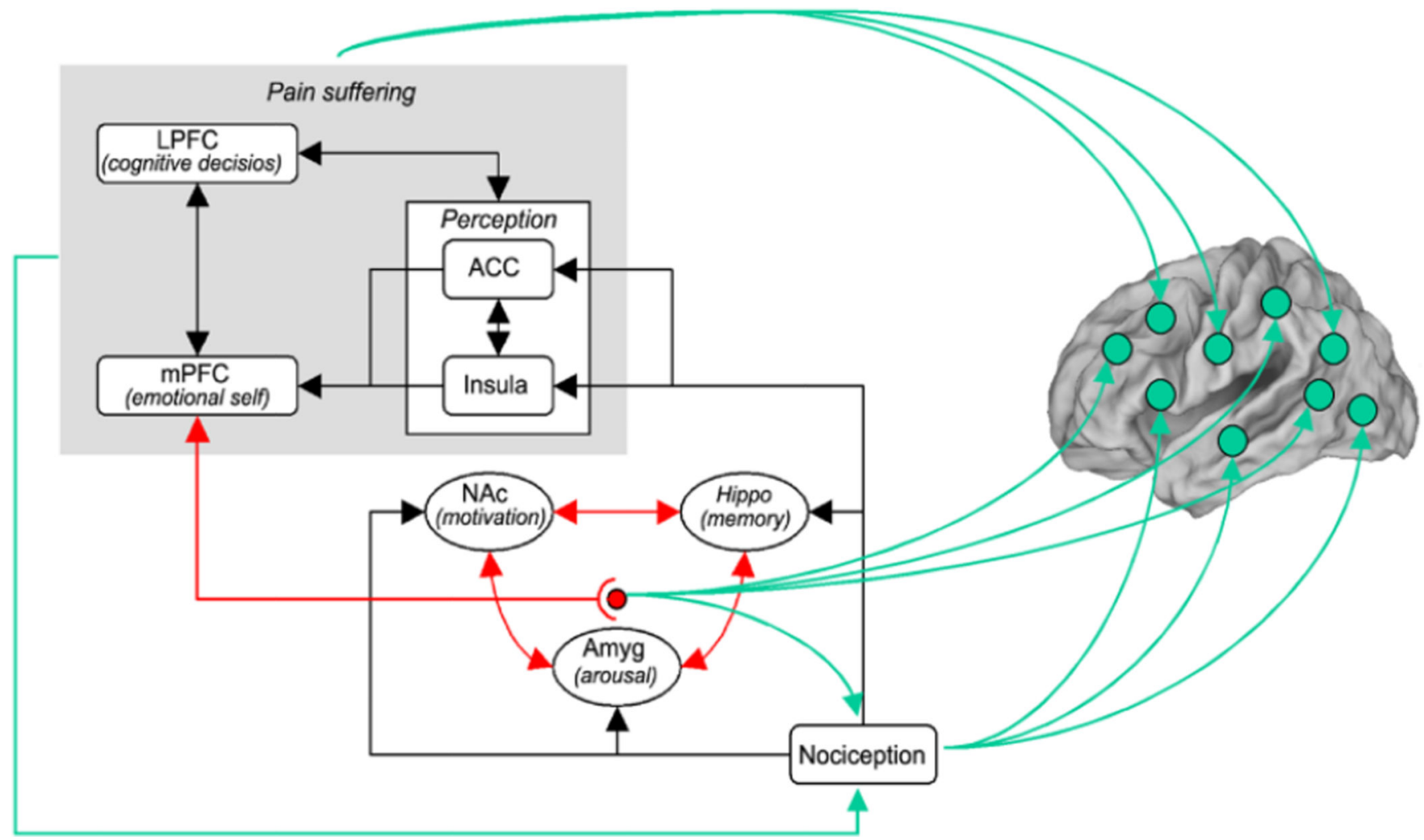

Figure 1.

A model regarding brain circuitry involved in the transition from acute to chronic pain. Nociceptive information, perhaps distorted by peripheral and spinal cord sensitization processes, impinges on limbic circuitry. The interaction of limbic circuitry with prefrontal processes determines the level at which a certain pain condition transitions to a more emotional state. The limbic circuitry also provides learning/modulation signals to the rest of the cortex inducing functional and anatomical distortions that reflect the suffering and coping strategies, adapted from (Apkarian et al., 2011). 

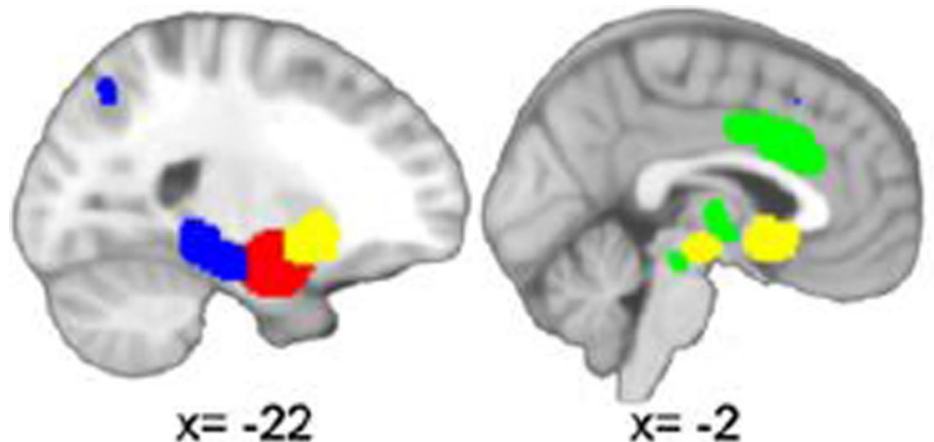

$x=-22$

$$
x=-2
$$
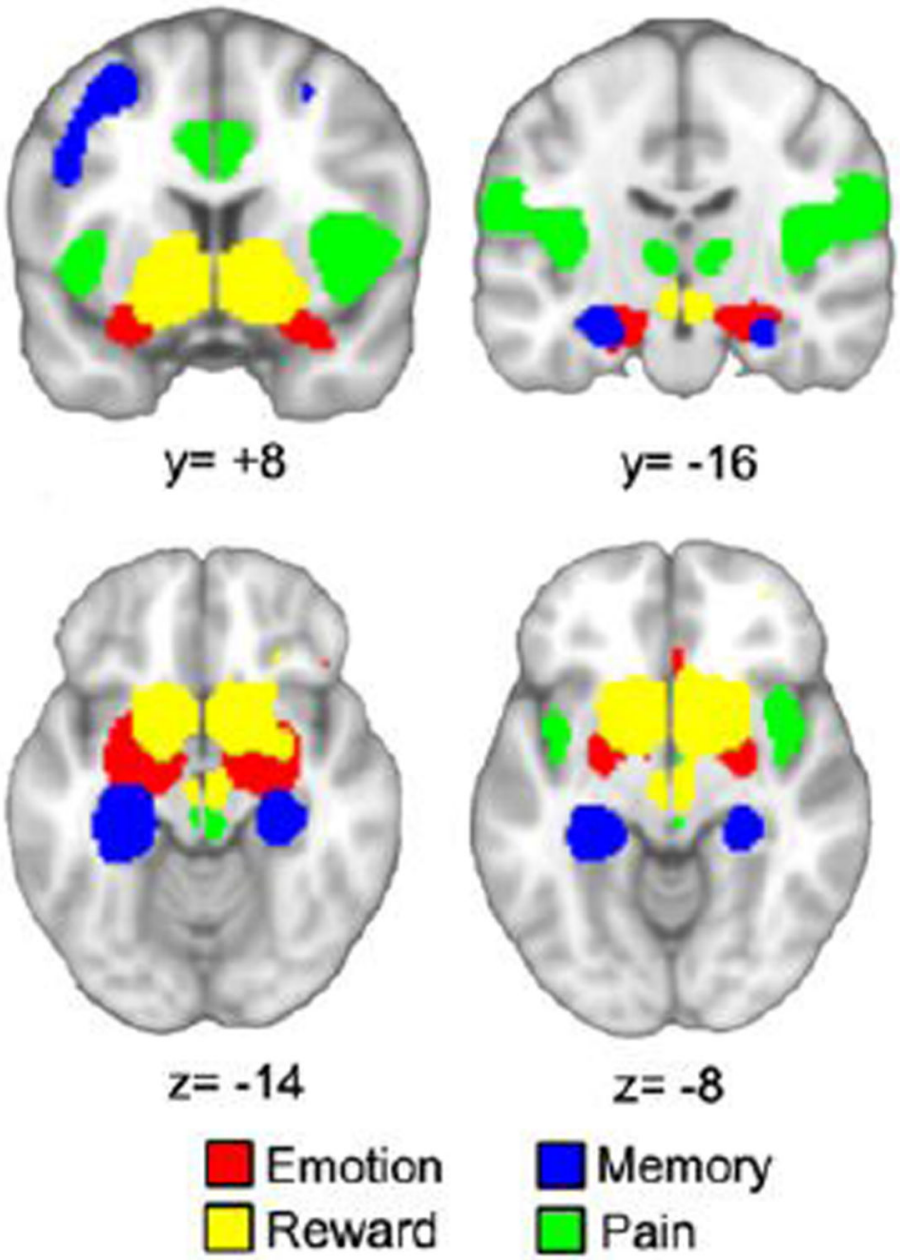

$$
y=-16
$$

Figure 2.

Brain activity meta-analysis maps derived from the Neurosynth tool (Yarkoni et al., 2011), for the four terms indicated. Only brain regions positively activated for each term are shown, with the appropriate color, when activity was thresholded at a z-value $>5.0$. At this threshold, "emotion" activates mainly bilateral amygdala and medial prefrontal cortex; "memory" activates bilateral hippocampus and parts of the posterior parietal and lateral prefrontal regions; "reward" activates bilateral nucleus accumbens, hypothalamus, and 
ventral tegmentum; "pain" activates bilaterally anterior cingulate, thalamus, periaqueductal gray, and insula. 